\title{
"Capacidad de autocuidado en adultos mayores para la realización de actividades instrumentales de la vida diaria Centro Poblado el Ron Cajaruro -2018"
}

\section{"Self-care ability in older adults for the performance of instrumental activities of the daily life of the Town Center the Ron Cajaruro -2018"}

\author{
Yshoner Antonio Silva Díaz ${ }^{1}$ Elito Mendoza Quijano²
}

\begin{abstract}
RESUMEN
El presente estudio fue de enfoque cuantitativo, nivel descriptivo, de tipo observacional, prospectivo, transversal y univariado, cuyo objetivo fue: Determinar la capacidad de autocuidado en adultos mayores para la realización de actividades instrumentales de la vida diaria del Centro Poblado el Ron Cajaruro -2018. La muestra estuvo conformada por de 86 adultos mayores para la recolección de datos se utilizó el método de la encuesta y la técnica del cuestionario, se utilizó el instrumento modificado de Lawton y Brody "Actividades instrumentales de la vida diaria" Presenta un coeficiente de reproductividad inter e intraobservador alto (0.94). En los resultados se encontró que del $100 \%$ de los adultos mayores el $80.2 \%$ es dependiente y el $19.8 \%$ es independiente, así mismo el sexo masculino posee el índice de mayor dependencia con $85.7 \%$, y el sexo femenino con una dependencia de $73 \%$, el grupo etario de 81 años a mas mostro un mayor índice de dependencia $100 \%$, y de 71-80 años menor dependencia con $73.7 \%$. En las dimensiones procedimentales se encontró que la responsabilidad de la medicación y medio de transporte fueron las dimensiones con mayor índice de dependencia con $83.7 \%$ y $75.6 \%$ respectivamente. Por lo tanto, se concluyó que la capacidad de autocuidado en adultos mayores del centro poblado el Ron para la realización de actividades instrumentales de la vida diaria es dependiente, más de la mitad de dicha población no practica su autocuidado.
\end{abstract}

Palabras clave: Adulto mayor, Capacidad de autocuidado, actividades instrumentales de la vida diaria.

\section{ABSTRACT}

The present study was of a quantitative approach, descriptive level, observational, prospective, cross-sectional, univariate, whose objective was: to Determine the self-care ability in older adults for the performance of instrumental activities of the daily life of the Town Center the Ron Cajaruro -2018. The sample consisted of 86 elderly adults for data collection using the method of the survey and the technique of the questionnaire, we used the instrument modified Lawton and Brody Instrumental Activities of daily living" Presents a coefficient of reproducibility inter-and intraobserver high (0.94). In the results, it was found that $100 \%$ of adults over the $80.2 \%$ is dependent, and $19.8 \%$ is independent, so the male has the rate of increased dependence with $85.7 \%$, and female sex, with a dependency of $73 \%$, the age group of 81 years but showed a higher dependency ratio $100 \%$, and 71-80 years less dependency with $73.7 \%$. In the dimensions of procedural, it was found that the responsibility of the medication and means of transport were the dimensions with a higher dependency ratio with $83.7 \%$ and $75.6 \%$ respectively. Therefore, it was concluded that the self-care ability in older adults of the town Centre Rum for the performance of instrumental activities of daily living is dependent, more than half of this population does not practice self-care.

Keywords: Older Adult, Capacity for self-care, instrumental activities of daily living.

'Doctor Ciencias de Enfermería. Docente principal a tiempo completo Universidad Nacional Toribio Rodríguez de Mendoza de Amazonas. ${ }^{2}$ Licenciado en Enfermería. Docente Universidad Nacional Toribio Rodríguez de Mendoza de Amazonas. Email: elito_mendoza@hotmail.com 


\section{INTRODUCCIÓN}

A nivel mundial el porcentaje de adultos mayores va aumentando en los diferentes países, así mismo el aumento de la esperanza de vida respectivamente tiende a sobresalir de 52 años en el quinquenio 19501955 hasta los 75 años en el quinquenio de 2010 2015, por otro lado, se estima que entre el 2000 y 2050 la proporción de las personas mayores de 60 años se duplicará, pasando del $11 \%$ al $22 \%$, en números cuantitativos pasará de 605 millones a 2000 millones respectivamente (OMS, 2017,p.4)

El proceso de envejecimiento de toda la población no es un fenómeno exclusivo de las sociedades modernas, es un proceso sistemático natural, considerado como uno de los logros más importantes de la humanidad, se transforma a la vez en un problema cuando las entidades y partes involucradas no es capaces de brindar soluciones adecuadas a las consecuencias derivadas del envejecimiento (Millán, 2010,p.10)

(MINSA, 2006) estableció los lineamientos para la Atención Integral de Salud de las Personas Adultas Mayores con la finalidad de brindar un marco referencial, así como un conjunto de orientaciones que puedan constituirse en la base de las acciones para la atención integral de salud, capaz de favorecer un envejecimiento saludable y autocuidado mejorando su estilo de vida, cada uno de ellos con un enfoque multidisciplinario, también existe cuatro lineamientos de política nacional esenciales; envejecimiento saludable, empleo previsión y seguridad social; participación e integración social, educación conciencia y cultura sobre el envejecimiento y la vejez, políticas que favorece el bienestar biopsicosocial de los adultos mayores (PLANPAM, 2013,p.11)

Es esencial recalcar que a pesar de los grandes esfuerzos del estado para salvaguardar la salud de las personas adultas mayores llevan un estilo de vida inadecuado provocando así la dependencia en las diferentes actividades instrumentales de la vida diaria provocando así en múltiples ocasiones enfermedades físicas, mentales, cognitivas y una deficiente calidad de vida.

En el Perú, la primera causa de mortalidad en los adultos mayores fueron las infecciones respiratorias $(23,39 \%)$ seguida por enfermedades isquémicas del corazón (22,74\%), enfermedades cerebro-vasculares $(18,88 \%)$ y el tumor maligno del estómago $(5,95 \%)$ que fue la primera causa de muerte por tumores (INEI, 2008, p.12)

En el Perú también se indica que más de 794.000 adultos mayores padecen de discapacidad, siendo esta etapa de la vida, en mayor porcentaje, el origen de las limitaciones de forma permanente para: moverse o caminar $(32,5 \%)$, ver aun usando anteojos $(51,3 \%)$, oír aún usan audífonos $(60,5 \%)$, entender o aprender $(39,1 \%)$, relacionarse con los demás por su pensamientos, sentimientos, emociones o conductas $(17,9 \%)$, hablar o comunicarse $(13 \%)$ (CONADIS, 2013,p.18)

En la región amazonas se evidencia que la mayor cantidad de adultos mayores padecen de dependencia es así que en los últimos años se identificó que el grado de dependencia y depresión entre los años 6079 es el $76 \%$ corresponde al sexo femenino y el $24 \%$ al sexo masculino; mientras que en el grupo de adultos mayores de 80 años el $52.2 \%$ corresponde al sexo femenino y el $47.8 \%$ al sexo masculino (DIRESA, 2017, p. 17)

El centro poblado el Ron cuenta con su Centro de Salud de nivel 1-3 teniendo como recurso humano el equipo básico de salud brindando así atenciones preventivo promocionales teniendo como población y grupo etario a las personas adultas mayores brindando una atención preventiva promocional todos los adultos mayores que acuden a dicho establecimiento el $70 \%$ de los casos son por problemas de caídas, complicación de su enfermedad hipertensión arterial, diabetes mellitus, por deficiente medicación, quemaduras (HIS, 2017,p.2)

Las Actividades Instrumentales de la vida Diaria son aquellas en las que la persona interacciona con el medio para mantener su independencia; permite la participación en la comunidad (transporte, compras, finanzas, uso del teléfono, toma de medicamentos, cocinar, que hacer doméstico y lavandería), todas estas actividades se ven comprometidas por los problemas que padece el adulto mayor generando muchas veces la dependencia es allí donde el profesional de enfermería juntamente con el equipo de salud de primer nivel de atención cumplen una labor importante de prevenir las enfermedades fomentar el autocuidado del adulto mayor mejorar su estilo de vida, la teoría de Dorothea Orem se encuentra conformada por tres teorías interrelacionadas entre sí : autocuidado, sistemas de enfermería y déficit de autocuidado.

Por toda la problemática se precisa plantear el siguiente problema de investigación. ¿Cuál es la capacidad de autocuidado en adultos mayores para la realización de actividades instrumentales de la vida diaria del Centro Poblado el Ron Cajaruro -2018" teniendo como objetivo: Determinar la capacidad de autocuidado en adultos mayores para la realización de actividades instrumentales de la vida diaria del Centro Poblado el Ron Cajaruro -2018" 


\section{MATERIAL Y MÉTODO}

El presente estudio tiene un enfoque cuantitativo, de nivel descriptivo de tipo: según la intervención del investigador es observacional, según la planificación de la toma de datos es prospectivo, según el número de ocasiones en que se medirá la variable de estudio es transversal, según el número de variables de interés es univariado (Supo, 2015,p. 2 - 18)

Población: 110 adultos mayores autovalentes que están registrados en el padrón nominado del centro de salud el Ron.

Muestra: 86 adultos mayores según la formula estadística de proporciones para la población finita, así mismo se realizó el muestreo probabilístico aleatorio simple, para las unidades muestrales.

Método: Encuesta

Técnica: Cuestionario (Canales, 1994, p. 275).

El instrumento de recolección de datos: se utilizó el instrumento modificado de Lawton y Brody "Actividades instrumentales de la vida diaria" que nos permitió identificar si ejecuta la actividad o no, consta de 8 ítems, "Usar el teléfono, ir de compras, preparar la comida, Realizar tareas del hogar, Lavar la ropa, Utilizar transportes, Controlar la medicación, Manejar el dinero" Presenta un coeficiente de reproductividad inter e intraobservador alto $(0.94)$.

\section{RESULTADOS}

Tabla 01: Capacidad de autocuidado

\begin{tabular}{lcc}
\hline & Capacidad de autocuidado \\
\hline & $\mathrm{fi}$ & $\%$ \\
\hline Dependiente & 69 & 80.2 \\
Independiente & 17 & 19.8 \\
\hline TOTAL & 86 & 100 \\
\hline
\end{tabular}

Fuente: Cuestionario de Lawton y Brody

En la tabla 01 se observa respecto a la capacidad de autocuidado del $100 \%$ (86) que fueron encuestados el $80.2 \%$ (69) es dependiente el $19.8 \%$ (17) es independiente.

Tabla 02: Capacidad de autocuidado según sexo.

\begin{tabular}{lcccc}
\hline \multicolumn{3}{c}{ Capacidad de autocuidado según sexo } \\
\hline Masculino & & \multicolumn{3}{c}{ Femenino } \\
\hline & fi & $\%$ & fi & $\%$ \\
\hline Dependiente & 42 & 85.7 & 27 & 73 \\
Independiente & 7 & 14.3 & 10 & 27 \\
\hline TOTAL & 49 & 100 & 37 & 100 \\
\hline
\end{tabular}

Fuente: Cuestionario de Lawton y Brody

En la tabla 02 se identifica que en el sexo masculino prevalece el índice de mayor dependencia con un porcentaje de $85.7 \%$ (42), mientras que en el sexo femenino prevalece el estado de dependencia con $73 \%$ (27); en cambio la independencia ocupa un menor porcentaje tanto en varones como en mujeres con $14.3 \%$ (7) y $27 \%$ (10) respectivamente.

Tabla 03: Capacidad de autocuidado según edad

\begin{tabular}{lcccccc}
\hline \multicolumn{5}{c}{ Capacidad de autocuidado según edad } \\
\hline & $\mathbf{6 0 - 7 0 a}$ & $\mathbf{7 1 - 8 0 a}$ & $\mathbf{8 1}$ a mas \\
\hline & fi & $\%$ & fi & $\%$ & fi & $\%$ \\
\hline Dependiente & 54 & 81.8 & 14 & 73.7 & 1 & 100 \\
Independiente & 12 & 18.2 & 5 & 26.3 & 0 & 0 \\
\hline TOTAL & 66 & 100 & 19 & 100 & 1 & 100
\end{tabular}

Fuente: Cuestionario de Lawton y Brody

En la tabla 03 se observa que existe una mayor prevalencia de dependencia en el grupo etario 81 años a mas con un porcentaje de $100 \%$ mientras que el grupo etario con dependencia mínima es el de 71-80 años con un porcentaje de $73.7 \%$ (7), por el contrario, el grupo etario con mayor independencia es el de 7180 años con un porcentaje de $26.3 \%$ (5) y el menor porcentaje de independencia concierne al grupo etario 60-70 años a más.

Tabla 04: Capacidad de autocuidado según dimensión procedimental.

\begin{tabular}{|c|c|c|c|c|c|c|c|c|c|c|c|}
\hline \multicolumn{12}{|c|}{ Capacidad de autocuidado según dimensión procedimental } \\
\hline & \multicolumn{2}{|c|}{$\begin{array}{c}\text { Capacidad } \\
\text { de utilizar } \\
\text { dinero }\end{array}$} & \multicolumn{2}{|c|}{$\begin{array}{l}\text { Responsable } \\
\text { de medicación }\end{array}$} & \multicolumn{2}{|c|}{$\begin{array}{l}\text { Medio de } \\
\text { transporte }\end{array}$} & $\begin{array}{l}\text { Lavado } \\
\text { de ropa }\end{array}$ & $\begin{array}{l}\text { Cuidado } \\
\text { de casa }\end{array}$ & $\begin{array}{l}\text { Preparar } \\
\text { comida }\end{array}$ & $\begin{array}{c}\text { Ir de } \\
\text { compras }\end{array}$ & $\begin{array}{l}\text { Usar el } \\
\text { teléfono }\end{array}$ \\
\hline & fi & $\%$ & fi & $\%$ & fi & $\%$ & $\%$ & $\%$ & $\%$ & fi $\%$ & $\%$ \\
\hline Depend. & 52 & 60.4 & 72 & 83.7 & 65 & 75.6 & 3844.2 & 5766.3 & 4147.7 & 5159.3 & 4046.5 \\
\hline Independ. & 34 & 39.6 & 14 & 16.3 & 21 & 24.4 & 4855.8 & 2933.7 & $45 \quad 52.3$ & 3540.7 & 4653.5 \\
\hline TOTAL & 86 & 100 & 86 & 100 & 86 & 100 & $86 \quad 100$ & $86 \quad 100$ & $86 \quad 100$ & $86 \quad 100$ & $86 \quad 100$ \\
\hline
\end{tabular}


En la tabla 04 se observa respecto a la capacidad de autocuidado según dimensión procedimental, del total $100 \%$ (86) en lo que concierne a responsabilidad de la medicación el $83.7 \%$ (72) son dependientes, y en medio de transporte el 75.6\% (65) son dependientes, ocupando el primer y segundo lugar de dependencia respectivamente, por el contario lavar ropa se identifica que el 55.8\% (48) son independientes, y el usar el teléfono el 53.5\% (46) son independientes siendo esta la dimensión de mayor independencia respectivamente.

\section{DISCUSIÓN}

La capacidad de autonomía de los adultos mayores en este trabajo de investigación se midió con el cuestionario de Lawton Brody concerniente a las actividades instrumentales de la vida diaria en donde abarca diferentes dimensiones procedimentales, de dependencia e independencia, el adulto mayor como se desenvuelve en la sociedad esas características instrumentales convive con su cotidianeidad, en donde esas actividades realizadas adecuadamente evidencian la independencia del adulto mayor.

Los determinantes sociales, conductuales, biológicos en donde se desarrolla el adulto mayor juegan un papel fundamental en el desenvolvimiento y desarrollo, pero son regularizados por políticas públicas del estado sobre la mejora de calidad de vida de esta población, a pesar de los grandes esfuerzos del gobierno para pacificar, promocionar y estabilizar el proceso de envejecimiento aún persiste la necesidad de mayor autonomía del adulto mayor en función a las actividades preventivas promocionales y potencializar los factores protectores.

En el presente trabajo de investigación en la tabla 01 se encontró que del $100 \%$ (86) de los adultos mayores que fueron encuestados en el centro poblado el Ron el 80.2\% (69) es dependiente para la realización de las actividades instrumentales de la vida diaria mientras que el $19.8 \%$ (17) es independiente, estos porcentajes tiene convergencia con la investigación de Garcilazo (2014) en donde encontraron como resultados en su investigación realizada en San juan de Miraflores que del total de adultos mayores encuestados 100\% (74), el 75,7\% (56) son dependientes y $24,3 \%$ (18) son independientes.

En la tabla 02 se muestra del total de adultos mayores encuestados en el centro poblado el Ron, fueron de sexo masculino (49) y de sexo femenino (37), de todos los encuestados de sexo masculino prevalece el índice de mayor dependencia con un porcentaje de 85.75 (42), mientras que de todos los encuestados del sexo femenino prevalece el estado de dependencia en un porcentaje de $73 \%$ (27); mientras que la independencia de actividades instrumentales ocupan un menor porcentaje tanto para varones como para mujeres en $14.3 \%$ (7) y $27 \%$ (10) respectivamente.

Evidentemente en el sexo masculino se mostró un mayor porcentaje de dependencia en esta investigación lo cual tiene similitud con la investigación de Millán (2010), quien encontró como resultado que del 100\% (141) adultos mayores encuestados el 50,5\% de los hombres y el 43,3\% de las mujeres no se cuida adecuadamente, y existe un déficit de autocuidado.

La edad como determinante y el proceso de envejecimiento de los adultos mayores juegan un papel fundamental en el desarrollo y evaluación de la dependencia funcional de las actividades instrumentales de la vida diaria por lo tanto la capacidad de autocuidado conforme avanza la edad se vuelve más vulnerable tal como se encontró en este trabajo de investigación en la tabla 03 se evidencia la mayor prevalencia de dependencia correspondiente al grupo etario 81 años a mas con un porcentaje de $100 \%$ mientras que el grupo etario con dependencia mínima es el de 71-80 años con un porcentaje de $73.7 \%(7)$.

Así mismo tiene similitud con la investigación de Camino (2011) lo cual concluye que "Los adultos mayores del centro de salud "Tablada de Lurín" en su mayoría poseen una capacidad de autocuidado poco aceptable en la prevención de caídas, de acuerdo a los factores de riesgo intrínsecos o relacionados con la edad y los factores extrínsecos relacionados con el entorno, lo que los pone en riesgo de sufrir caídas y sus posibles consecuencias físicas, psicológicas y socio familiares alterando el mantenimiento de la salud, bienestar y sobre todo la independencia y la autonomía

Las actividades instrumentales de la vida diaria se midieron por dimensiones con la finalidad de identificar en que dimensión o actividades el adulto mayor cuenta con mayor dependencia e independencia respectivamente, es así que en la tabla 04 se identificó que del total de encuestados 100\% (86) en lo que concierne a la responsabilidad de la medicación el $83.7 \%$ (72) son dependientes, y en medio de transporte el 75.6\% (65) son dependientes, ocupando el primer y segundo lugar de dependencia respectivamente, claramente evidencia altos índices de dependencia estos resultados es alarmante porque el autocuidado del adulto mayor no se está evidenciando.

Estos resultados según dimensión procedimental 
convergen con la investigación de Garcilazo (2014) quien encontró que las capacidades para ir de compras y preparar la comida y responsabilidad de la medicación son las áreas más comprometidas con el $43,2 \%, 41,9 \%$ y $54,1 \%$ de dependencia respectivamente, claramente en la dimensión responsabilidad sobre la medicación en ambos estudios ocupan el primer lugar por lo que es importante analizar los factores externos que posibilitan una mejora en relación indirecta y directa con las personas adultas mayores.

Estas cifras son alarmantes el centro poblado el Ron cuenta con adultos mayores que no realizan un autocuidado, sino que requieren ayuda de otras personas para la realización de las actividades instrumentales, es importante que los profesionales de la salud que laboran en dicha comunidad eduquen y pongan en marcha como equipo multidisciplinario el cuidado enseñanza hacia el autocuidado proveyendo y fortaleciendo los diferentes factores protectores externos e internos de la sociedad donde se desenvuelven los adultos mayores.

La teoría del autocuidado según Dorotea Oren enfatiza la clave el manejo la intervención del profesional enfermero para brindar un cuidado y ser facilitador en el proceso de independencia de las personas, teniendo en cuenta un ambiente biopsicosocial efectivo, aire adecuado, soporte alimentario adecuado, suministro de agua adecuado proceso de eliminación, descanso y confort, interacción social adecuado, bienestar humano y formación de grupos, todo ello para la mejora continua.

\section{CONCLUSIONES}

La capacidad de autocuidado en adultos mayores del centro poblado el Ron para la realización de actividades instrumentales de la vida diaria es dependiente, más de la mitad de dicha población no practica su autocuidado.

En el centro poblado el Ron el mayor número y porcentaje de adultos mayores dependientes para la realización de actividades instrumentales de la vida diaria pertenecen al sexo masculino mientras que lo femenino aparece en un porcentaje menor.

En el centro poblado el Ron el grupo etario que posee mayor dependencia es el de 81 años y el grupo etario de mayor independencia es el de 71-80 años.

Los adultos mayores del centro poblado el Ron en las dimensiones responsabilidad de la medicación y en medio de transporte son dependientes, ocupando el primer y segundo lugar de dependencia respectivamente.

\section{VI .REFERENCIAS}

Canales (1994) "Metodología de la investig ación." México: Limusa S.A. de C.V.

Camino, S. (2011) “Capacidad de autocuidado en la prevención de caídas que tienen los adultos mayores Centro de Salud Tablada de Lurín" (Tesis de licenciatura) Universidad Nacional Mayor de San Marcos, Lima, Perú Recuperado de: file://C:/ARTICULOS/para $\%$ 20articuloooo/Camino_sm.pdf

CONADIS (2013) "Informe del consejo Nacional de Discapacidad" recuperado de: (http://www.inei.gob.pe/web/Biblioinei/Bol etinFlotante.asp?file=16034.pdf

DIRESA. (2017) "Informe sobre prevalencia de dependencia y depresión en la región: Amazonas-Perú" Recuperado de: https://www.ugr.es/ recfpro/rev61COL5.pd $\mathrm{f}$

Garcilazo (2014) "Capacidad de autocuidado del adulto mayor para realizar las actividades instrumentales de la vida diaria. en un centro de salud de San Juan de Miraflores 2014" (Tesis de licenciatura), Universidad Nacional Mayor de San Marcos, Lima Perú. Recuperado de: file://C:/ARTICULOS/par a\%20articuloooo/importanteeee $\% 20$ baseee. pdf

HIS (2017) "Reporte de atenciones de las Enfermedades prevalentes del centro poblado el Ron" recuperado de: http:/ /dge.gob.pe/portal/Asis/indreg/asis_amazo nas.pdf

INEI (2008) "Perfil Sociodemográfico del Perú" recuperado de:http://www.inei. gob.pe/Ane xos/libro.pdf

MINSA (2006) "Norma técnica de Salud para la Atención Integral de Salud de las personas adultas mayores" Recuperado de: ftp://ftp2.minsa.gob.pe/normaslegales/2006 /RM529-2006.pdf

Millán (2010) “Evaluación de la capacidad y percepción de autocuidado del adulto mayor en la comunidad." (Tesis de licenciatura), Universidad Habana Cuba. Habana, Cuba. Recuperado de: http: //scielo. Sld .cu/scielo .php?script $=$ sci_arttext\&pid $=\mathrm{S} 0864031920$ $10000400007 \& \operatorname{lng}=\mathrm{es}$

OMS (2017) "Datos y cifras: envejecimiento y s a l ud" $\mathrm{r}$ e c u p e r a d o d e : http://www.who.int/mediacentre/factsheets/ 
fs404/es/. Acceso el día 14 de abril del año 2018.

PLANPAM (2013) "Informe del plan nacional de personas adultas mayores" recuperado de: http://www.mimp.gob.pe/files/mimp/espec ializados/boletines_dvmpv/cuaderno_5_dv mpv.pdf

Supo (2015) "Seminarios de investigación cientifica" recuperado de: file://C:Users/PC/downloads/107josc3a9supo-cc3b3mo-empezar-una-tesis.pdf 